\title{
Uma análise da manufatura aditiva aplicada ao planejamento urbano sustentável: revisão de aplicações, desafios e potencialidades
}

O crescimento populacional e a expansão urbana originaram diversos desafios, como a eficiência do gerenciamento urbano, considerado obstáculo para alcançar a resiliência urbana. Uma das soluções para enfrentar esse e outros desafios é a integração do planejamento urbano baseado em conceitos, como a sustentabilidade e a manufatura aditiva. Diante deste contexto, este artigo objetivou desenvolver o 'estado da arte' da manufatura aditiva, e ainda, abordar seu surgimento e avanços no setor urbano, com a apresentação dos desafios e suas potencialidades. Dessa forma, esta revisão de literatura, baseada nos estudos de Büyüközkan et al. (2018), ocorreu em periódicos científicos nas bases de dados ScienceDirect, Scopus, Web of Science e IEEE Xplore, utilizando como descritores: 'manufatura aditiva', 'impressão 3D', 'planejamento urbano', 'gestão urbana', e 'sustentabilidade', todos com foco em inovações no setor da construção civil. Apresentou-se o fenômeno urbano e as alternativas empregadas para o planejamento urbano, bem como, as contribuições da manufatura aditiva para o meio ambiente atreladas ao planejamento urbano. Em seguida, ressaltou-se as patentes registradas que contribuíram para os avanços e as técnicas reconhecidas ate hoje. E por fim, foi apresentado um breve histórico da aplicação de construções 3D, os desafios e suas potencialidades. Portanto, foi possível perceber que as contribuições mostradas nesta revisão podem fomentar a sustentabilidade e contribuir para o planejamento urbano sustentável.

\section{An analysis of additive manufacturing applied to sustainable urban planning: review of applications, challenges and potentialities}

Population growth and urban expansion gave rise to several challenges, such as the efficiency of urban management, considered an obstacle to achieving urban resilience. An option to face this and other challenges is the integration of urban planning based on concepts such as sustainability and additive manufacturing. Given this context, this article aimed to develop the 'state of the art' of additive manufacturing, and also address its emergence and advances in the urban sector, with the presentation of challenges and its potential. Thus, this literature review, based on the studies by Büyüközkan et al. (2018), took place in scientific journals in the databases ScienceDirect, Scopus, Web of Science and IEEE Xplore, using as descriptors: 'additive manufacturing', '3D printing ', 'urban planning', 'urban management', and 'sustainability', all focusing on innovations in the civil construction sector. The urban phenomenon and the alternatives used for urban planning were presented, as well as the contributions of additive manufacturing to the environment linked to urban planning. Then, the registered patents that contributed to the advances and techniques recognized until today were highlighted. And finally, a brief history of the application of 3D constructions, the challenges and thei potential was presented. Therefore, it was possible to see that the contributions shown in this review can foster sustainability and contribute to sustainable urban planning.

Keywords: 3D printing; Innovation; Sustainability.

Topic: Desenvolvimento, Sustentabilidade e Meio Ambiente

Reviewed anonymously in the process of blind peer.
Received: 02/07/2021

Approved: $28 / 07 / 2021$
Maicon Douglas Leles da Silva (id

Universidade Cesumar, Brasil

http://lattes.cnpq.br/1264373180181917

http://orcid.org/0000-0002-7176-6033

maiconleles@gmail.com

Gabriela Burali Bergamasco (iD

Universidade Cesumar, Brasil

http://lattes.cnpq.br/2106749483950453

http://orcid.org/0000-0002-0370-1844

gabrielabergamasco25@gmail.com

Lilian Keylla Berto (iD

Universidade Cesumar, Brasil

http://lattes.cnpq.br/8201764678168686

http://orcid.org/0000-0003-0343-9433

luciana.rezende@unicesumar.edu.br
Luciana Cristina Soto Herek Rezende (iD

Universidade Cesumar, Brasil

http://lattes.cnpq.br/1158006438170059

http://orcid.org/0000-0001-9677-4139

uciana.rezende@unicesumar.edu.br
Referencing this:

SILVA, M. D. L.; BERGAMASCO, G. B.; BERTO, L. K.; REZENDE, L. C. S. H. Uma análise da manufatura aditiva aplicada ao planejamento urbano sustentável: revisão de aplicações, desafios e potencialidades. Revista Ibero Americana de Ciências Ambientais, v.12, n.7, p.328-341, 2021. DOI: http://doi.org/10.6008/CBPC2179-6858.2021.007.0030 


\section{INTRODUÇÃO}

Com o crescimento populacional houve consequentemente a expansão urbana, pois, a maior parte disponibilidade de empregos, seja público ou privado, encontram-se nas áreas urbanas. Esse fenômeno urbano atraiu a maior quantidade de pessoas nos grandes centros. Nesse cenário, um dos grandes desafios do século XXI é a eficiência do gerenciamento urbano, com foco na saúde, bem-estar, acessibilidade e recursos naturais (GARCIA et al., 2020; GAVRILIDIS, et al., 2020).

Esse desafio apresentado pode ter cunho ambiental, social e econômico, e se torna um obstáculo para alcançar a resiliência nas áreas urbanas (BUSH et al., 2019). Além disso, a expansão urbana não planejada, como consequência do crescimento urbano, tem gerado catástrofes ambientais, como os deslizamentos de terras, alterações climáticas, inundações urbanas, aumento na quantidade de precipitações, impacto socioeconômico, destruição de parte da infraestrutura e até mesmo habitações (MATEOS et al., 2020).

Atualmente, neste cenário, destaca-se a sustentabilidade que por sua vez tem sido muito aplicada e tem ganhado destaque em todo o mundo, em especial em cidades com o desenvolvimento muito rápido. 0 foco principal do planejamento sustentável é promover recursos sociais e econômicos de forma igualitária e durável, assim como ambientes urbanos com qualidade (LA ROSA et al., 2020; LIANG et al., 2020).

Como as cidades têm se transformado em algo extremamente complexo, as técnicas do planejamento urbano tradicional têm se tornado de difícil aplicação, dessa forma, é necessário ajustes e alterações para alcançar a sustentabilidade (LA ROSA et al., 2020; LIANG et al., 2020). Uma das soluções para enfrentar esse e outros desafios é com a integração do planejamento urbano baseado em conceitos que podem contribuir diretamente para o aumento da resiliência urbana (BUSH et al., 2019).

Dessa forma, a sustentabilidade e o desenvolvimento sustentável são importantes para a redução dos impactos ambientais, principalmente no setor da construção civil (KARJI et al., 2019). E uma das inovações neste setor que se mostra alinhada ao tripé da sustentabilidade, pois possui vantagens sociais, econômicas e ambientais é a manufatura aditiva (SAKIN et al., 2017; HAGER et al., 2016).

No contexto mundial, a manufatura aditiva tem mostrado destaque em diversas áreas, dentre elas estão a biomedicina, a engenharia mecânica, a aeronáutica, a eletrônica (GUO et al., 2019) e a engenharia civil (LU et al., 2019). Esta tecnologia, mais conhecida como impressão 3D, é utilizada para construir objetos tridimensionais por uma determinada técnica. Dentre as técnicas existentes para essa finalidade, destacamse a estereolitografia (SLA), modelagem por deposição fundida (FDM), escrita direta à tinta (DIW), sinterização seletiva a laser (SLS) (GUO et al., 2019), Contour Crafting (SAKIN et al., 2017).

Cada técnica possui características diferentes, como o material empregado, área de aplicação, dentre outros. Por exemplo, a técnica Contour Crafting foi desenvolvida para aplicação no setor urbano e tem como principal característica o sistema de construção por meio da deposição do material cimentício realizado camada por camada para a construção de casas, prédios, pontes, dentre outras. Ainda, o processo de automatização da impressão desta técnica permite a construção in situ, ou seja, realizada no próprio local 
(HAGER et al., 2016).

Diante desse contexto, este trabalho busca responder às seguintes questões de pesquisa: Quais são as contribuições da manufatura aditiva para o meio ambiente atreladas ao planejamento urbano? Quais são os desafios e potencialidades da manufatura aditiva no setor urbano? Para responder a essas questões, o objetivo deste artigo foi desenvolver o 'estado da arte', com a finalidade de descrever o estágio atual da manufatura aditiva. Tem ainda por objetivo abordar os avanços da manufatura aditiva e seu surgimento no setor da construção civil, com a apresentação dos desafios e suas potencialidades.

\section{METODOLOGIA}

Esta pesquisa é caracterizada como qualitativa e possui caráter descritivo. Foi baseada nos estudos de Büyüközkan et al. (2018), por meio da leitura crítica, seletiva e interpretativa de artigos em periódicos científicos (internacionais e nacionais), dos anos de 2016 até o primeiro semestre de 2020, utilizando as seguintes bases de dados online: ScienceDirect, Scopus, Web of Science e IEEE Xplore.

A busca de artigos científicos foi realizada durante todo o primeiro semestre de 2020 (janeiro-junho). Neste período foram analisadas publicações utilizando os descritores: 'manufatura aditiva', 'impressão 3D', 'planejamento urbano', 'gestão urbana', e 'sustentabilidade', todos com foco em inovações no setor da construção civil.

Seguindo este raciocínio, este trabalho apresenta o desenvolvimento do 'estado da arte', com a finalidade de descrever o surgimento da manufatura aditiva na construção civil, abordando seus desafios e suas potencialidades.

Para isso, foram propostas as seguintes questões de partida: 1. Quais são as contribuições da manufatura aditiva para o meio ambiente atreladas ao planejamento urbano?; 2. Quais são os desafios e potencialidades da manufatura aditiva no setor urbano?

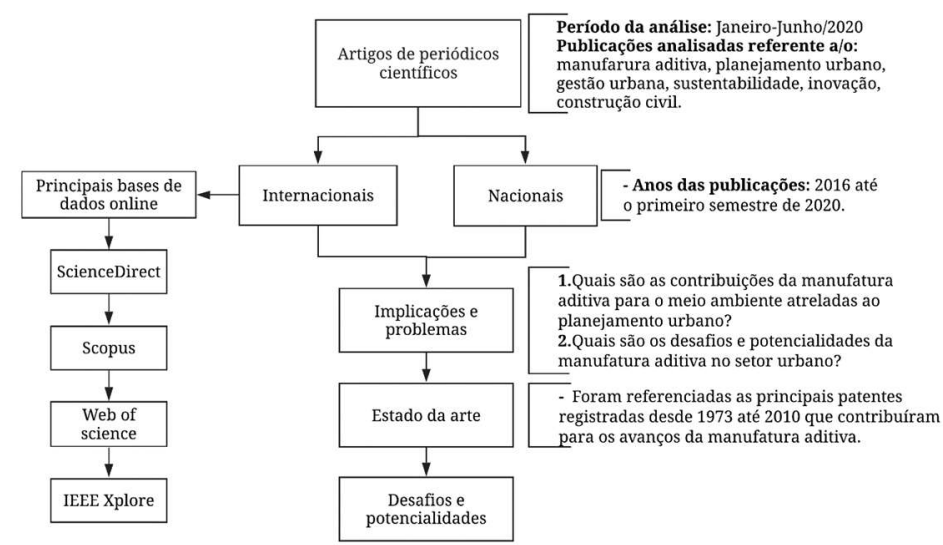

Figura 1: Metodologia utilizada na pesquisa baseada em Büyüközkan et al. (2018)

Deste modo, em um primeiro momento foi apresentado um breve histórico do conceito 'manufatura aditiva' e das construções 3D com destaque nas tendências de conceitualização. Em um segundo momento e última parte, apresentou-se os desafios e potencialidades identificados após a leitura crítica dos artigos.

Para o 'estado da arte' foram referenciadas também as principais patentes registradas que marcaram 
os avanços da manufatura aditiva, desde 1973 até 2010. A fim de tornar compreensível o caminho metodológico adotado nesta pesquisa, a Figura 1 apresenta as etapas desenvolvidas neste trabalho, baseando-se nos problemas de pesquisa e na metodologia de Büyüközkan et al. (2018).

\section{DISCUSSÃO TEÓRICA}

Nesta seção, a revisão de literatura é desenvolvida por meio da apresentação do fenômeno urbano e das alternativas empregadas para o planejamento urbano, bem como, os novos conceitos adotados no contexto da gestão urbana, com enfoque na sustentabilidade e nos benefícios da manufatura aditiva aplicada ao setor da construção civil.

Em um segundo momento, é descrito o 'estado da arte' da manufatura aditiva com as principais patentes registradas referentes aos dispositivos e técnicas que contribuíram para os avanços dessa tecnologia. Em seguida, tem-se um breve histórico da aplicação das construções 3D. E por fim, são apresentados os desafios e potencialidades da manufatura aditiva no setor urbano.

\section{Planejamento urbano e novos conceitos}

O fenômeno da urbanização e o processo de expansão urbana causam como efeitos a vulnerabilidade socioambiental e os riscos socioambientais. Em contrapartida, a resiliência socioecológica urbana surge como uma condição de capacidade adaptativa de recuperar ou adquirir um equilíbrio no sistema urbano (CABRAL et al., 2019).

Além da busca pelo aumento das áreas verdes nos centros urbanos, a resiliência urbana tem sido adotada no planejamento urbano. É considerada um ajuste na política urbana e se refere à capacidade de ser adaptar frente às mudanças. Também, tem crescido muito as iniciativas nacionais e internacionais, mas requer longo prazo e diversas perspectivas para sua implementação. A interligação entre resiliência e a sustentabilidade busca melhores caminhos para as transições urbanas (BUSH et al., 2019).

Numa análise global, um dos documentos mais atuais de iniciativa global é conhecido como Agenda 2030, que integra a sustentabilidade às cidades com foco nas questões sociais, ambientais e econômicas. Esta agenda apresenta os princípios e propósitos da Carta das Nações Unidas e possui um plano de ações composto por dezessete Objetivos de Desenvolvimento Sustentável (ODS) e 169 metas (LASLETT et al., 2020).

A agenda 2030, que tem chamado a atenção de pesquisadores e o atendimento aos ODS, se mostra uma das formas encontradas para mitigar os impactos ambientais negativos (OMER et al., 2019). Como exemplo, no contexto urbano, o fortalecimento do planejamento urbano com infraestrutura verde busca conceituar os espaços naturais conectados aos ambientes urbanos, de modo a fomentar os objetivos dos Objetivos de Desenvolvimento Sustentável (ODS), tem-se mostrado uma alternativa que vem em contrapartida com a redução de espaços naturais e o crescimento de áreas não planejadas nos centros urbanos (GARCIA et al., 2020).

Em outra análise, o crescimento populacional e a concentração de pessoas nos grandes centros, representa um forte mecanismo para inovação cultural e social que atribui para a sociedade novos 
comportamentos, economias e tecnologias (MARANGHI et al., 2020).

Nesse sentido surgiram diversos conceitos que abordam novos aspectos para as cidades e suas construções, como cidades inteligentes (CHAMOSO et al., 2020; LASLETT et al., 2020; LAZZARETTI et al., 2019), cidades sustentáveis (JALAEl et al., 2020), infraestrutura verde (MAROPO et al., 2019), construções sustentáveis (OLIVEIRA et al., 2019), indústria 4.0, Impressão 3D (DALLASEGA et al., 2018), Building Information Model (BIM), manufatura aditiva (SAKIN et al., 2017; HAGER et al., 2016) e Smart Manufacturing System (PHUYAL et al., 2020).

Cada vez mais as cidades têm buscado estratégias sustentáveis, a fim de melhorarem a eficiência energética em edifícios, a fim de trazerem inclusão social, proteção ambiental e viabilidade financeira (BOSCH et al., 2019). Outra contribuição é a inserção da tecnologia em conjunto com os novos métodos na indústria da construção que possam contribuir para que as políticas públicas e organizações consigam alcançar os ODS (SAIEG et al., 2018).

Dentre as termologias atuais, destaca-se o conceito de cidade inteligente, que é baseado em qualidade de vida com a inserção de tecnologias de informação e comunicação (LASLETT et al., 2020; LAZZARETTI et al., 2019), de modo a promover interações entre as cidades e a gestão pública (LAZZARETTI et al., 2019). Dessa forma, as cidades inteligentes promovem um estilo de vida mais sustentável. Além disso, a infraestrutura, inovação e tecnologia são os componentes que tornam as cidades inteligentes eficientes e autossuficientes (CHAMOSO et al., 2020).

Diante de todo o avanço tecnológico, é notório que as tecnologias se tornam tão importantes no contexto das cidades, inclusive para o monitoramento da qualidade ambiental, social e econômica (LASLETT et al., 2020), conhecidos como tripé da sustentabilidade. Os profissionais das áreas de arquitetura e de engenharia têm mostrado interesse nessas dimensões de obras, projetos e processos mais eficientes para o setor da construção civil (ARANGO et al., 2019), promovendo, desta forma, as construções mais sustentáveis para alcançarem o conceito de cidades sustentáveis (JALAEI et al., 2020).

O conceito de cidades sustentáveis se diferencia dos demais, pois não pode ser aplicado linearmente e está baseado no tripé da sustentabilidade. Ou seja, para alcançá-lo as cidades devem ser sustentáveis em todas as áreas. Nesse sentido, as cidades sustentáveis se apoiam em três pilares com base no foco a Agenda 2030, englobando pessoas, lucro e planeta (LASLETT et al., 2020). Dentre os ODS da Agenda 2030, merece destaque o de número 11, que se refere às cidades e comunidades sustentáveis (LASLETT et al., 2020; MARANGHI et al., 2020).

O cumprimento aos objetivos sustentáveis resultará em esforços positivos com relação aos aspectos de bem-estar, eficiência energética e hídrica; também poderá promover o consumo responsável e a produção mais limpa. Além disso, o atendimento dos ODS pode mitigar os problemas relacionados as mudanças climáticas, o que torna a infraestrutura resiliente e as cidades sustentáveis (OMER et al., 2019).

A sustentabilidade urbana tem se tornado cada vez mais relevante e o seu alcance um dos maiores desafios atuais (MARANGHI et al., 2020). É considerada uma ótima reação para os problemas urbanos recentes de crescimento populacional (LASLETT et al., 2020), impacto ambiental negativo e a perda da 
biodiversidade (OPOKU, 2019).

Essa sustentabilidade urbana se mostra evidente nas iniciativas ambientais globais para proporcionar uma melhor interação entre os componentes que envolvem a humanidade e a natureza (OPOKU, 2019), bem como estratégias para alcançar a economia verde e o desenvolvimento sustentável (LASLETT et al., 2020).

Nos estudos de Laslett et al. (2020) por meio do método matemático de agregação aritmética, geométrica e harmônica foi possível verificar que a sustentabilidade é um forte princípio de desempenho a ser alcançado e avaliado em ranking das cidades e, desta forma, mostrou que a sustentabilidade está cada vez mais visível e evidenciada no contexto urbano.

Dentro do contexto urbano surge a construção civil, que está em constante processo de evolução e busca atender as novas tendências e conceitos. Por exemplo, o conceito infraestrutura verde que surge em oposição à infraestrutura convencional, com o uso de métodos e tecnologias mais sustentáveis com finalidade de recuperar áreas verdes degradadas, eficiência e economia (MAROPO et al., 2019).

Nota-se que desde a concepção dos novos projetos e construções, a sustentabilidade é um dos fatores determinantes, e é ainda mais evidenciada no processo de certificações de construções sustentáveis, como a LEED (Liderança em Energia e Design Ambiental) (OLIVEIRA et al., 2019).

A LEED é uma das certificações mais usuais e conhecidas (JALAEI et al., 2020; SHEALY, 2016), que avalia com pontuação as construções verdes por categorias que inclui espaço sustentável, localização e transporte, eficiência da água, energia e atmosfera, materiais e recursos, qualidade do ambiente construído, inovação, prioridade regional e processo integrativo (JALAEI et al., 2020).

Os níveis de pontuação se dividem em prata com 40 a 49 pontos, ouro de 60 a 79 pontos e platina com 80 pontos ou mais (GREER et al., 2019). Desta forma, os edifícios sustentáveis possuem uma alta pontuação da certificação LEED em comparação com os edifícios tradicionais devido aos benefícios ambientais, econômicos e sociais (SHEALY, 2016).

Desta forma, a implementação e os investimentos de ferramentas, tecnologias e treinamento profissional, alinhado à gestão urbana, aos incentivos governamentais e setores privados podem promover desenvolvimento sustentável, com destaque no setor da construção civil (SAIEG et al., 2018).

Nos últimos anos, a transformação digital e as tecnologias inteligentes se transformaram em pilares para as indústrias das futuras gerações, como a recente indústria 4.0 e a sociedade 5.0 e têm impulsionado os avanços aos sistemas de manufatura aditiva, como, por exemplo, o conceito Smart Manufacturing System, utilizado para se referir aos sistemas inteligentes da manufatura aditiva e se mostra uma tendência colaborativa e criativa para diversos setores, o que tem potencializado a área de engenharia e arquitetura (PHUYAL et al., 2020).

É notório que os projetos que integram novos métodos, como o BIM - definido por Sakin et al. (2017), como o gerenciamento e compatibilização de projetos sustentados por tecnologias digitais que avaliam todo o ciclo de vida de uma construção. Referido método está se fortalecendo com a manufatura aditiva (PHUYAL et al., 2020) em conjunto com as tecnologias de suporte, como a pré-fabricação, realidade virtual e técnicas de impressão 3D, que por consequência, podem melhorar a qualidade dos gerenciamentos nas organizações 
e diminuir retrabalhos, alterações no projeto, tempo e custos econômicos (DING et al., 2019).

Nos estudos de Jalaei et al. (2020), o BIM foi integrado para avaliar a pontuação para alcançar um desempenho de construção com base na certificação LEED. Dessa forma, os projetos que possuírem a plataforma BIM integrada a novos métodos, como a manufatura aditiva no setor da construção civil, terão maior tendência para alcançar a eficiência energética, a otimização no design, a redução de custos (SAKIN et al., 2017), por consequência, sucesso no planejamento e processo de execução complexo (MECHTCHERINE et al., 2019).

\section{Manufatura aditiva e meio ambiente}

A manufatura aditiva aplicada no setor da construção civil é capaz de imprimir estruturas, e para isso, exige condições de processos para a construção que controlam os equipamentos utilizados para o gerenciamento sofisticado, baseados no BIM, que por sua vez integra o complexo planejamento e processo de execução, com todas as informações geométricas e específicas dos projetos (MECHTCHERINE et al., 2019).

Atualmente, a evolução da manufatura aditiva originou o conceito Smart Manufacturing System, que consiste em proporcionar um sistema de fabricação inteligente por meio de um gerenciamento com foco em controle, monitoramento, digitalização e eficiência. Com isso, esse conceito utiliza os princípios das cidades inteligentes, como o uso de tecnologias de informação e comunicação incorporadas ao modelo da indústria 4.0 (PHUYAL et al., 2020).

A transformação digital originalmente aplicada à manufatura aditiva na era da Indústria 4.0 ainda é considerada lenta e seu potencial ainda não foi totalmente explorado no gerenciamento da cadeia de suprimentos, no entanto, traz mudanças ao setor da construção. A realidade virtual e a interação humanocomputador também são vistas como os principais componentes da indústria 4.0 que permitem ambientes de construção digitalizados (DALLASEGA et al., 2018).

Já os componentes a serem explorados pela manufatura aditiva e seu conceito Smart Manufacturing System são o gerenciamento do ciclo de vida do produto, gerenciamento da cadeia de suprimentos, design para fabricação e montagem, sistema de manufatura flexível e reconfigurável, ciclo de inovação rápida, dentre outros (PHUYAL et al., 2020).

O ciclo de vida do produto se torna diretamente aliado a sustentabilidade, pois esta pode ser aplicada no setor urbano com a inserção de processos construtivos limpos, que agregam aspectos sociais, como saúde e maior produtividade de construções (SHEALY, 2016), possuindo, dessa forma, vantagens relacionadas ao tripé da sustentabilidade. $O$ desenvolvimento sustentável no setor da construção civil é importante para a redução dos impactos ambientais e tem mostrado avanços com métodos e técnicas mais sustentáveis (KARJI et al., 2019).

Alguns métodos de construção foram evoluídos com a incorporação de conceitos mais sustentáveis e eficientes, tais como Building Information Model (BIM), construção enxuta (SAIEGET et al., 2018) e manufatura aditiva (SAKIN et al., 2017; HAGER et al., 2016), o que proporciona uma redução de resíduos e o aumento no controle de processos e segurança dos trabalhadores (SAIEG et al., 2018), que por consequência, 
fomenta o desenvolvimento sustentável.

Os métodos utilizados na manufatura aditiva podem contribuir para maior eficiência, maior liberdade para formas geométricas e redução de resíduos (LU et al., 2019). Dessa forma, a manufatura aditiva aplicada para a construção de edificações é uma tecnologia promissora e sustentável. Além disso, a técnica de manufatura aditiva mais utilizada recentemente no setor da construção civil é a Contour Crafting (SAKIN et al., 2017).

O sistema de construção desta técnica é por meio da deposição do material, geralmente, compósito de matriz cimentícia, realizada camada por camada e com a aplicação da automatização do processo de construção que pode ser utilizada para a construção in situ, ou seja, realizada no próprio local (HAGER et al., 2016).

Nas últimas décadas a manufatura aditiva se destaca na área da construção civil (LU et al., 2019), pois se mostra alinhada ao tripé da sustentabilidade, devido as vantagens sociais, econômicas e ambientais (Tabela 1), relacionadas à possibilidade da impressão de elementos no próprio local (SAKIN et al., 2017), a sua capacidade de programação de máquinas para a realização de tarefas que pode reduzir os acidentes de trabalho (HAGER et al., 2016) e sua contribuição para a redução dos impactos ambientais (SAKIN et al., 2017). Além disso, o material de matriz cimentícia pode conter materiais que possam ser reciclados, tais como os resíduos de blocos cerâmicos e de fibras de celulose (EL-SAYEGH et al., 2020).

Tabela 1: Benefícios na utilização da manufatura aditiva em construções em relação ao tripé da sustentabilidade.

\begin{tabular}{|c|c|c|}
\hline $\begin{array}{l}\text { Aspectos em relação ao tripé da } \\
\text { sustentabilidade }\end{array}$ & Benefícios em comparação com os métodos tradicionais & Referência \\
\hline Aspectos ambientais & $\begin{array}{l}\text { Redução da geração de resíduos da construção civil } \\
\text { Redução da poluição no meio ambiente } \\
\text { Promove a reciclagem com a incorporação de resíduos } \\
\text { Reduz desperdícios }\end{array}$ & $\begin{array}{l}\text { EL-SAYEGH et al. } \\
\text { (2020) } \\
\text { LU et al. (2019) } \\
\text { SAKIN et al. (2017) } \\
\text { HAGER et al. (2016) }\end{array}$ \\
\hline Aspectos sociais & $\begin{array}{l}\text { Redução na geração de poluentes atmosféricos } \\
\text { Processos mais controlados e mais seguros } \\
\text { Evita riscos à saúde }\end{array}$ & $\begin{array}{l}\text { HAGER et al. (2016) } \\
\text { PHUYAl et al. } \\
\text { (2020) }\end{array}$ \\
\hline Aspectos econômicos & $\begin{array}{l}\text { Redução de custos de transporte, armazenagens de insumo, } \\
\text { matérias-primas e energia } \\
\text { Otimização do tempo } \\
\text { Maior precisão } \\
\text { Aumento na produtividade }\end{array}$ & $\begin{array}{l}\text { SAKIN et al. (2017) } \\
\text { PHUYAL et al. } \\
\text { (2020) }\end{array}$ \\
\hline
\end{tabular}

\section{Estado da arte da manufatura aditiva}

O novo pensamento de fabricação de produtos surgiu com os novos métodos e patentes, primeiramente, visto como prototipagem rápida (SCHMIDT et al., 2017). Ciraud (1973) registrou patente de seu método e dispositivo capaz de transformar o pó metálico em um corpo sólido por meio de feixes de raio laser. Outro registro de patente foi de Swainson (1977) que desenvolveu um método e aparelho que recria a geometria do objeto em uma figura tridimensional de forma aditiva por meio de feixes cruzados de radiação.

Uma das patentes mais semelhante com os processos de modelagem de sinterização a laser atuais foi a registrada pelo Housholder (1981), na qual o objeto se forma com o emprego de partículas fusíveis de laser que são aplicadas seletivamente camadas por camada no material. 
No mesmo ano, Kodama (1981) desenvolveu sua técnica de manufatura aditiva que transforma um líquido polimérico em sólido por meio da aplicação em camadas com raios ultravioletas. Anos depois, essa técnica foi aprimorada e denominada como estereolitografia pelo registro da patente de Hull (1986). No final da década de 80, Deckard (1989) registrou patente de seu método denominado por sinterização seletiva a laser, semelhante ao de Hull (1986), contudo, foi investigado no material em pó.

A partir de então, o desenvolvimento da manufatura aditiva foi impulsionado pela alta produtividade da indústria 4.0 (PHUYAL et al., 2020) e pelo surgimento de softwares CAD (computer-aided designs) e CAM (computer-aided manufacturing), os quais incentivaram a modelagem em três dimensões (3D) de peças mecânicas de forma mais eficiente e rápida (RAJAGURU et al., 2020).

Um grande destaque da manufatura aditiva foi com o registro da patente de Crump (1992), que definiu o método e aparelho da técnica de deposição de filamento fundido. Nesta patente foi sugerido o emprego dos softwares CAD e CAM para a produção de objetos tridimensionais por meio de materiais, como os termoplásticos. Nesta técnica o material é depositado por camadas em uma temperatura prédeterminada ao longo dos eixos denominados ' $X$ ', ' $Y$ ' e ' $Z$ ' e é solidificado em uma base com outra taxa controlada de temperatura.

Nos últimos anos, essa tecnologia apresenta rápido crescimento no contexto mundial e tem mostrado destaque em diversas áreas, dentre elas estão a biomedicina, a engenharia mecânica, a aeronáutica, a eletrônica (GUO et al., 2019), a medicina veterinária (WILHITE et al., 2019), a engenharia civil (LU et al., 2019), dentre outras.

A redução de custos, desenvolvimento e aprimoramento de técnicas são os que tornam a manufatura aditiva cada vez mais acessível e aplicada em diversas áreas no contexto urbano (WILHITE et al., 2019). Hoje em dia, existem diversas técnicas para a aplicação da manufatura aditiva, por exemplo, estereolitografia (SLA), modelagem por deposição fundida (FDM), escrita direta à tinta (DIW), sinterização seletiva a laser (SLS) (GUO et al., 2019), Contour Crafting (SAKIN et al., 2017).

A análise comparativa entre as diferentes técnicas de impressão 3D, volta-se para aspectos fundamentais, tais como materiais utilizados, precisão, acabamento, resistência mecânica. Por exemplo, na técnica Contour Crafting são necessários materiais eficientes para a fabricação de uma matriz cimentícia que tenha capacidade de impressão para a execução de uma edificação, peças arquitetônicas e estruturais, além das propriedades reológicas e mecânicas apropriadas para serem utilizadas (HAGER et al., 2016).

\section{Breve histórico da aplicação de construções 3D no setor urbano}

A construção civil está em constante processo de evolução e com a manufatura aditiva utilizando o método Contour Crafting houve um novo capítulo da tecnologia no setor construtivo (HAGER et al., 2016). A saber, a manufatura aditiva no setor da construção civil foi criada pelo Dr. Behrokh Khoshnevis e denominada Contour Crafting (SALET et al., 2018). Khoshnevis (1996) registrou a patente do aparelho e método de manufatura aditiva voltados para a utilização de materiais de construção. Khoshnevis (2010) registrou outra patente 'Deployable contour crafting' que descreve sobre o sistema de portíco acoplado em um veículo para 
a execução da construção de um edifício.

Ao decorrer dos anos a impressão de peças ou até mesmo edifícios por completos são construídos em diversas partes do mundo, principalmente por meio da técnica Contour Crafting (Tabela 2).

Tabela 2: Avanços na manufatura aditiva aplicada no setor da construção civil.

$\begin{array}{ll}\text { Ano } & \text { Avanços } \\ 2008 & \text { Criação da primeira impressora 3D em escala real pela empresa WinSun na China. }\end{array}$

2013 Impressão de lotes de peças (pré-fabricadas) para a realização de dez casas, pela empresa WinSun na China, em sua fábrica por meio de uma impressora com dimensão de 6 × 10 × 40m (altura x largura x comprimento).

Referência

EL-SAYEGH et

al. (2020)

EL-SAYEGH et

al. (2020)

GHAFFAR et

al. (2018)

HAGER et al.

(2016)

Após o transporte das peças impressas em 2013 pela empresa chenesa, foram executadas as casas por meio da montagem das peças, incluindo os componentes como portas, janelas e coberturas, em apenas 24 horas.

Construção do castelo Andy Rudenko's garden - em Minnesota, Estados Unidos - considerada a primeira estrutura de construção in situ, ou seja, realizada no próprio local.

GHAFFAR et

al. (2018)

HAGER et al.

(2016)

EL-SAYEGH et

al. (2020)

Ainda, a empresa WinSun realizou na China a construção com a aplicação da manufatura aditiva de uma mansão de $1100 \mathrm{~m}^{2}$ e do primeiro prédio com dimensão de 6,6 x 40 × 10m (altura x comprimento x largura).

Em Amsterdã, Holanda, foi projetada e realizada pela DUS Architects a construção da Urban Cabin, traduzido para o português, cabine urbana. Em 24 horas por meio da técnica FDM (Fused Deposition Modeling) esta construção de material polimérico, segundo Rajan et al. (2018), bioplástico sustentável e reciclável, possui área útil de $8 \mathrm{~m}^{2}$. Impressão de um hotel com dimensões 12,5 x 10,5 x 4m (altura x comprimento x largura), realizado por Andrey Rudenko. Essa obra impressa teve duração interrupta de 100 horas com a utilização de uma matriz cimentícia com bom desempenho e ligação entre camadas.

BUCHANAN et

al. (2019)

GHAFFAR et

al. (2018)

RAJAN et al.

(2018)

HAGER et al

(2016)

EL-SAYEGH et

A empresa chinesa WinSun também realizou o primeiro escritório impresso de $250 \mathrm{~m}^{2}$ em Dubai, conhecido como 'Office of the Future', traduzindo para o português, como o escritório do futuro. As peças foram produzidas com a técnica de manufatura aditiva na China e a execução do projeto em Dubai em 17 dias. Houve um aumento na produtividade e na eficiência da obra que reduziu em quase metade de custos com mão de obra e resíduos de construção.

2016 Construção da primeira ponte realizada com a impressão 3D, localizada no parque urbano Castilla-La Mancha, na Espanha. A impressão total compõe oito peças foi realizada pelo Institute of Advanced Architecture, em Catalunha, Barcelona. A obra da ponte com dimensão de 12 metros de comprimento e 1,75m de largura teve duração de dois meses.

al. (2020)

BUCHANAN et

al. (2019)

GHAFFAR et

al. (2018)

RAJAN et al.

(2018)

SAKIN et al.

(2017)

Em Nantes, na França foi realizada a construção da Yhnova ${ }^{\mathrm{TM}}$, uma habitação social de $95 \mathrm{~m}^{2}$, com aplicação de manufatura aditiva para a impressão de paredes de concreto com preenchimento externo e interno de espuma de polímero, com duração de 54 horas de trabalho por meio de um robô de impressora 3D.

Finalização da execução de uma ponte de concreto protendido com peças impressas em laboratório para a obra de renovação de uma ciclovia na vila Germert, na Holanda, com dimensão de $8 \mathrm{~m}$ x 3,5 m de comprimento e largura, respectivamente, e vão livre de $6,5 \mathrm{~m}$. O processo de impressão teve duração de 48 horas, utilizando dois sistemas de impressão com troca a cada duas horas, devido ao tempo de endurecimento do concreto ser de 2 a 3 horas, o que

2017 permitiu evitar falhas de impressão ou entupimento de material no sistema.

Na Europa foi impresso em Copenhague pela empresa 3D Printhuset um prédio com um design que enfatiza liberdade geométrica e redução de linhas retas.

A empresa lançou a residência Apis Cor House de $38 \mathrm{~m}^{2}$ - localizada na capital da Rússia - executada no próprio local em 24, utilizou tendas para isolar toda a obra da neve, no qual foi a solução a limitação do concreto geopolimérico poder ser impresso acima de $5^{\circ} \mathrm{C}$.

A empresa holandesa $C y B e$ também desenvolveu uma impressora pela técnica Contour Crafting. Realizou obras impressas referentes a uma casa de $80 \mathrm{~m}^{2}$ na Arábia Saudita, uma ponte na Holanda e um laboratório.

EL-SAYEGH et al. (2020)

BUCHANAN et

al. (2019)

FURET et al.

(2019)

GHAFFAR et

al. (2018)

POULLAIN et

al. (2018)

SALET et al.

(2018)

SAKIN et al.

(2017)

Construção da maior ponte em concreto impressa em Xangai, na China, desenvolvida pela Universidade de Arquitetura

2019 de Tsinghua, com o comprimento de 86 ft (pés) com duração de impressão de 450 horas. A análise de econômica mostrou uma redução de custos de aproximadamente um terço.

EL-SAYEGH et al. (2020)

\section{Desafios da manufatura aditiva e suas potencialidades no setor urbano}

O conceito manufatura aditiva aplicada na construção tem impulsionado as diferentes áreas das engenharias e arquitetura a trabalharem de forma interdisciplinar de forma a atenderem as demandas e desafios da Contour Crafting. Dessa forma, os processos inteligentes e automação se mostram essenciais para superar esses desafios, desde a concepção do design até a impressão em escala real (MECHTCHERINE et al., 2019), como, por exemplo, o emprego de softwares avançados CAD/CAM em conjunto com a plataforma BIM e certificação LEED (JALAEI et al., 2020). 
Além de enfrentar esses desafios, a manufatura aditiva se mostra promissora para superar barreiras ambientais e sociais e contribuir para o planejamento urbano sustentável, visto que os conceitos aplicados no setor urbano que integram a construção da resiliência urbana baseadas na sustentabilidade são soluções para enfrentar os impactos ambientais, as mudanças climáticas, além de trazer desenvolvimento econômico e social (BUSH et al., 2019).

No contexto atual, a sustentabilidade aplicada nas construções de edificações se tornou um ponto chave no desenvolvimento das cidades sustentáveis (JALAEI et al., 2020). Dessa forma, é notória as grandes contribuições da manufatura aditiva para o meio ambiente atrelado ao planejamento urbano. Também, há de se considerar que um dos objetivos da manufatura aditiva se mostra alinhado ao planejamento urbano, pois ambos têm a capacidade de remodelar espaços com o gerenciamento e o desenvolvimento da infraestrutura e serviços (BUSH et al., 2019).

A manufatura aditiva aplicada no setor urbano se destaca por apresentar um alto potencial construtivo, inclusive em larga escala, com previsões de atender demandas atuais dos projetos arquitetônicos e estruturais (MECHTCHERINE et al., 2019), e futuras, a saber, nos Emirados Árabes Unidos há previsões de impressão de casas até o ano de 2030 (RAJAN et al. 2018).

Cabe ressalvar que essa tecnologia já foi aplicada na implantação de casas, prédios comerciais e habitacionais (SAYEGH et al., 2020), estruturas complexas como um castelo (HAGER et al., 2016) e pontes (BUCHANAN et al., 2019; SALET et al., 2018), e até para a implantação de habitação social, investigada nos estudos de Poullain et al. (2018) e Furet et al. (2019).

À medida que as pesquisas vêm aprimorando seus equipamentos, métodos construtivos e materiais, os pontos de fraqueza se dissolvem (LASLETT et al., 2020) e o planejamento das cidades apresentam evolução (GARCIA et al., 2020). Dessa forma, o setor da construção tem adotado métodos mais eficientes e com menor impacto ambiental, e por consequência, contribuição a sustentabilidade (ARANGO et al., 2019), ao ODS de número 11, cujo foco é 'cidades e comunidades sustentáveis' (MARANGHI et al., 2020).

Daí o crescimento das investigações científicas, com a finalidade de aprimorar a manufatura aditiva aplicada no setor urbano, conhecida como a técnica Contour Crafting, principalmente, ao desenvolvimento de materiais eficientes para serem aplicados a esta técnica. Os desafios no setor da construção civil em relação aos fatores estimulantes para o desenvolvimento de materiais à base de cimento são principalmente a satisfação na obtenção de desempenhos e aplicações, novos métodos teóricos e experimentais, e eliminação de novas preocupações com os aspectos ambientais (HAMIDI et al., 2019).

Portanto, o desenvolvimento de compósitos de matriz cimentícia que tenham propriedades satisfatórias, além da capacidade de impressão para serem utilizadas na técnica Contour Crafting torna-se um desafio (HAGER et al., 2016).

A resistência à deformação do material está relacionada às propriedades de extrudabilidade e construtibilidade, primordiais para o processo 3D utilizado no setor construtivo, porém, entram em conflito quando se trata da parte prática. Enquanto na extrudabilidade há necessidade de que ocorra fluidez no início do preparo do material, na construtibilidade, é necessário que o material apresente uma rigidez inicial, pois 
isso será responsável pela estabilidade na construção das camadas do mesmo (WENG et al., 2019).

Tem-se importância, dessa maneira, as pesquisas que buscam o desenvolvimento de materiais cimentícios para uso na manufatura aditiva com foco na sustentabilidade, podendo ser incorporado materiais de reforço, que melhorem as propriedades mecânicas e as de impressão (LU et al., 2019).

Um reforço adicionado aos materiais compósitos pode ter resultados satisfatórios nas patologias apresentadas nas construções (SOLTAN et al., 2018) e é um método construtivo mais sustentável (KARJ et al., 2019), onde a manufatura aditiva possui indícios de uma produção mais limpa, e consequentemente, pode trazer uma minimização dos efeitos relacionados às mudanças climáticas, promovendo infraestruturas resilientes e cidades mais sustentáveis (OMER et al., 2019).

\section{CONCLUSÕES}

Por meio desta pesquisa foi possível perceber as contribuições da manufatura aditiva para o ambiente atreladas ao planejamento urbano, como o gerenciamento sofisticado, a fabricação inteligente, a redução de custos e de resíduos. Além disso, essas contribuições mostradas nesta revisão podem fomentar a sustentabilidade e o desenvolvimento das cidades sustentáveis.

No que tange o objetivo deste trabalho em desenvolver o 'estado da arte' da manufatura aditiva, pode-se dizer que, este novo pensamento de construção tridimensional surgiu desde a década de 70 por meio de registros de patentes com a apresentação de métodos e dispositivos que posteriormente se tornaram técnicas que tem ganhado espaço em diversas áreas, como a Contour Crafting, aplicada no setor urbano para construções de casas, prédios, pontes, dentre outras.

O desenvolvimento deste trabalho também apresentou os desafios e potencialidades desta técnica que surgem desde a concepção inicial de projetos com o auxílio de softwares avançados CAD/CAM até o aprimoramento de equipamentos, técnicas e o desenvolvimento de compósitos de matriz cimentícia. Por fim, foi ressaltado que essa tecnologia pode ser promissora para superar barreiras ambientais e sociais de forma a contribuir positivamente para o planejamento urbano sustentável.

\section{REFERÊNCIAS}

ARANGO, D. C.; JARAMILLO, S. B.; MONSALVE, P. A.; HERNÁNDEZ, A. V.; BOTERO, L. F. B.. Relationships between lean and sustainable construction: Positive impacts of lean practices over sustainability during construction phase. Journal of Cleaner Production, v.234, p.1322-1337, 2019. DOI: http://doi.org/10.1016/j.jclepro.2019.05.216

BOSCH, F. P.; CERVERA, C.; YSA, T.. Key aspects of building retrofitting: Strategizing sustainable cities. Journal of Environmental Management, v.248, e.109247, 2019. DOI: http://doi.org/10.1016/j.jenvman.2019.07.018

BUCHANAN, C.; GARDNER, L.. Metal 3D printing in construction: A review of methods, research, applications, opportunities and challenges. Engineering Structures, v.180, p.332-348, 2019. DOI:

http://doi.org/10.1016/i.engstruct.2018.11.045
BUSH, J.; DOYON, A.. Building urban resilience with naturebased solutions: How can urban planning contribute?. Cities, v.95, e.102483, 2019. DOI:

http://doi.org/10.1016/i.cities.2019.102483

BÜYÜKÖZKAN, G.; GÖÇER, F.. Digital Supply Chain: Literature review and a proposed framework for future research. Computers in Industry, v.97, p.157-177, 2018. DOI: http://doi.org/10.1016/j.compind.2018.02.010

CABRAL, L. N.; CÂNDIDO, G. A.. Urbanização, vulnerabilidade, resiliência: relações conceituais e compreensões de causa e efeito. Urbe. Revista Brasileira de Gestão Urbana, v.11, e.20180063, 2018. DOI: http://doi.org/10.1590/2175- 3369.011.002.A008

CIRAUD, P.. Verfahren und Vorrichtung zur Herstellung beliebiger Gegenstände aus beliebigem schmelzbarem 
Material. German patent application DE, 2263777, priority filed December 28th, 1971, published July 5th. 1973.

CRUMP, S. S.. Apparatus and method for creating threedimensional objects, U.S. Patent n.5.121-329. Washington: U.S. Patent and Trademark Office, 1992.

CHAMOSO, P.; GONZÁLEZ-BRIONES, A.; LA PRIETA, F.; VENYAGAMOORTHY, G. K.; CORCHADO, J. M.. Smart city as a distributed platform: Toward a system for citizen-oriented management. Computer Communications, v.152, p.323332, 2020. DOI:

http://doi.org/10.1016/j.comcom.2020.01.059

DALLASEGA, P.; RAUCH, E.; LINDER, C.. Industry 4.0 as an enabler of proximity for construction supply chains: $A$ systematic literature review. Computers in Industry, v.99, p.205-225, 2018. Dol:

http://doi.org/10.1016/j.compind.2018.03.039

DECKARD, C. R.. Method and apparatus for producing parts by selective sintering, U.S. Patent No. 4,863,538.

Washington: U.S. Patent and Trademark Office, 1989.

DING, Z., LIU, S.; LIAO, L.; ZHANG, L.. A digital construction framework integrating building information modeling and reverse engineering technologies for renovation projects. Automation in Construction, v.102, p.45-58, 2019. DOI: http://doi.org/10.1016/i.autcon.2019.02.012

EL-SAYEGH, S.; ROMDHANE, L.; MANJIKIAN, S.. A critical review of 3D printing in construction: benefits, challenges, and risks. Archives of Civil and Mechanical Engineering, v.20, n.2, p.1-25, 2020. DOI: http://doi.org/10.1007/s43452020-00038-w

FURET, B.; POULLAIN, P.; GARNIER, S.. 3D printing for construction based on a complex wall of polymer-foam and concrete. Additive Manufacturing, v.28, p.58-64, 2019. DOI: http://doi.org/10.1016/j.addma.2019.04.002

GARCIA, M. J.; CHRISTIEN, L.; GARCÍA-ESCALONA, E.; GONZÁLEZ-GARCÍA, C.. Sensitivity of green spaces to the process of urban planning. Three case studies of Madrid (Spain). Cities, v.100, e.102655, 2020. DOI: http://dx.doi.org/10.1016/j.cities.2020.102655

GAVRILIDIS, A. A.; POPA, A. M.; NITA, M. R.; ONOSE, D. A.; BADIU, D. L.. Planning the 'unknown': Perception of urban green infrastructure concept in Romania. Urban Forestry \& Urban Greening, e.126649, 2020. DOI: http://dx.doi.org/10.1016/j.ufug.2020.126649

GHAFFAR, S. H.; CORKER, J.; FAN, M.. Additive manufacturing technology and its implementation in construction as an eco-innovative solution. Automation in Construction, v.93, p.1-11, 2018. DOI:

http://doi.org/10.1016/j.autcon.2018.05.005

GREER, F.; CHITTICK, J.; JACKSON, E.; MACK, J.; SHORTLIDGE, M.; GRUBERT, E.. Energy and water efficiency in LEED: How well are LEED points linked to climate outcomes?. Energy and Buildings, v.195, p.161-167, 2019. DOI: http://doi.org/10.1016/i.enbuild.2019.05.010

GUO, H.; LV, R.; BAl, S.. Recent advances on 3D printing graphene-based composites. Nano Materials Science, v.1, n.2, p.101-115, 2018. DOI:

http://doi.org/10.1016/i.nanoms.2019.03.003

HAGER, I.; GOLONKA, A.; PUTANOWICZ, R.. 3D printing of buildings and building components as the future of sustainable construction. Procedia Engineering, v. 151, p.292-299, 2016. DOI:

http://doi.org/10.1016/j.proeng.2016.07.357

HAMIDI, F.; ASLANI, F.. Additive manufacturing of cementitious composites: Materials, methods, potentials, and challenges. Construction and Building Materials, v.218, p.582-609, 2019. DOI:

http://doi.org/10.1016/j.conbuildmat.2019.05.140

HOUSHOLDER, R. F.. Molding process, U.S. Patent No. 4,247,508. Washington: U.S. Patent and Trademark Office, 1981.

HULL, C. W.. Apparatus for production of three-dimensional objects by stereolithography, U.S. Patent No. 4,575,330. Washington: U.S. Patent and Trademark Office, 1986.

JALAEI, F.; JALAEI, F.; MOHAMMADI, S.. An integrated BIMLEED application to automate sustainable design assessment framework at the conceptual stage of building projects. Sustainable Cities and Society, v.53, e.101979, 2020. DOI: http://doi.org/10.1016/j.scs.2019.101979

KARJI, A.; WOLDESENBET, A.; KHANZADI, M.; TAFAZZOLI, M.. Assessment of Social Sustainability Indicators in Mass Housing Construction: A Case Study of Mehr Housing Project. Sustainable Cities and Society, v.50, e.101697, 2019. DOI: http://doi.org/10.1016/j.scs.2019.101697

KHOSHNEVIS, B.. Additive fabrication apparatus and method, U.S. Patent No. 5,529,471. Washington: U.S. Patent and Trademark Office, 1996.

KHOSHNEVIS, B.. Deployable contour crafting, U.S. Patent No. 7,814,937. Washington: U.S. Patent and Trademark Office, 2010.

KODAMA, H.. Automatic method for fabricating a threedimensional plastic model with photo-hardening polymer. Review of Scientific Instruments, v.52, n.11, p.1770-1773, 1981. DOI: http://doi.org/10.1063/1.1136492

La Rosa, D.; Pappalardo, V.. Planning for spatial equity-A performance based approach for sustainable urban drainage SYSTEMS. Sustainable Cities and Society, v.53, e.101885, 2020. DOI: http://dx.doi.org/10.1016/j.scs.2019.101885

LASLETT, D.; URMEE, T.. The effect of aggregation on city sustainability rankings. Ecological Indicators, v.112, e.106076, 2020. DOI: http://doi.org/10.1016/i.ecolind.2020.106076

LAZZARETTI, K.; SEHNEM, S.; BENCKE, F. F.; MACHADO, H. P. V.. Cidades inteligentes: insights e contribuições das pesquisas brasileiras. Urbe. Revista Brasileira de Gestão Urbana, v.11, e20190118, 2019. DOI: http://doi.org/10.1590/2175-3369.011.e20190118

LIANG, Y.; DU, M.; WANG, X.; XU, X.. Planning for urban life: A new approach of sustainable land use plan based on transit-oriented development. Evaluation and Program 
Planning, v.80, e.101811, 2020. DOl: http://doi.org/10.1016/i.evalprogplan.2020.101811

LU, B; WENG, Y.; LI, M.; QIAN, Y.; LEONG, K. F.; TAN, M. J.; QIAN, S.. A systematical review of 3D printable cementitious materials. Construction and Building Materials, v.207, p.477-490, 2019. DOI:

http://doi.org/10.1016/j.conbuildmat.2019.02.144

MARANGHI, S.; PARISI, M. L.; FACCHINI, A.; RUBINO, A.; KORDAS, O.; BASOSI, R.. Integrating urban metabolism and life cycle assessment to analyse urban sustainability. Ecological Indicators, v.112, e.106074, 2020. DOI: http://doi.org/10.1016/j.ecolind.2020.106074

MAROPO, V. L. B.; MORAIS, E. E.; NUNES, A. C.; SILVEIRA, J. A. R.. Planejamento urbano sustentável: um estudo para implantação de infraestrutura verde no Bairro Bancários, João Pessoa-PB, Brasil. Urbe. Revista Brasileira de Gestão Urbana, v.11, e.20180005, 2019. DOI:

http://doi.org/10.1590/2175-3369.011.002.A009

MATEOS, R. M.; LÓPEZ-VINIELLES, J.; POYIADJI, E.; TSAGKAS, D.; SHEEHY, M.; HADJICHARALAMBOUS, K.; GAUERT, C.. Integration of landslide hazard into urban planning across Europe. Landscape and Urban Planning, v.196, e.103740, 2020. DOI:

http://dx.doi.org/10.1016/j.landurbplan.2019.103740

MECHTCHERINE, V.; NERELLA, V. N.; WILL, F.; NÄTHER, M.; OTTO, J.; KRAUSE, M.. Large-scale digital concrete construction: CONPrint3D concept for on-site, monolithic 3D-printing. Automation in Construction, v.107, e.102933, 2019. DOI: http://doi.org/10.1016/j.autcon.2019.102933

OLIVEIRA, J. C.; FARIA, A. C.. Impacto econômico da construção sustentável: a reforma do Estádio do Mineirão. Urbe. Revista Brasileira de Gestão Urbana, v.11, e.20180031, 2019. DOI: http://doi.org/10.1590/21753369.011.001.A006

OMER, M. A.; NOGUCHI, T.. A conceptual framework for understanding the contribution of building materials in the achievement of Sustainable Development Goals (SDGs). Sustainable Cities and Society, v.52, e.101869, 2019. DOI: http://doi.org/10.1016/j.scs.2019.101869

OPOKU, A.. Biodiversity and the built environment: Implications for the Sustainable Development Goals (SDGs). Resources, Conservation and Recycling, v.141, p.1-7, 2019. DOI: http://doi.org/10.1016/j.resconrec.2018.10.011

PHUYAL, S.; BISTA, D.; BISTA, R.. Challenges, opportunities and future directions of smart manufacturing: a state of art Review. Sustainable Futures, v.2, e.100023, 2020. DOI: http://doi.org/10.1016/j.sftr.2020.100023

POULLAIN, P.; PAQUET, E.; GARNIER, S.; FURET, B.. On site deployment of 3D printing for the building construction-The case of YhnovaTM. MATEC Web of Conferences, v.163, e.01001, p.1-10, 2018. DOI: http://doi.org/10.1051/matecconf/201816301001

RAJAGURU, K.; KARTHIKEYAN, T.; VIJAYAN, V.. Additive manufacturing-State of art. Materials Today: Proceedings, v. 21, p.628-633, 2020. DOI: http://doi.org/10.1016/j.matpr.2019.06.728

RAJAN, A.; AKRE, V.; NASSIRI, N.; ALALI, A. H.; SABT, Z. B.. 3D Printing of Buildings in UAE: Success and Failure factors. Fifth HCT Information Technology Trends (ITT), p.368-372, 2018. DOI: http://doi/org/10.1109/CTIT.2018.8649541

SAIEG, P.; SOTELINO, E. D.; NASCIMENTO, D.; CAIADO, R. G. G.. Interactions of building information modeling, lean and sustainability on the architectural, engineering and construction industry: a systematic review. Journal of Cleaner Production, v.174, p.788-806, 2018. DOI: http://doi.org/10.1016/i.jclepro.2017.11.030

SAKIN, M.; KIROGLU, Y. C.. 3D Printing of Buildings: Construction of the Sustainable Houses of the Future by BIM. Energy Procedia, v.134, p.702-711, 2017. DOI: http://doi.org/10.1016/j.egypro.2017.09.562

SALET, T. A. M.; AHMED, Z. Y.; BOS, F. P.; LAAGLAND, H. L. M.. Design of a 3D printed concrete bridge by testing. Virtual and Physical Prototyping, v.13, n.3, p.222-236, 2018. DOI: http://doi.org/10.1080/17452759.2018.1476064

SCHMIDT, M.; MERKLEIN, M.; BOURELL, D.; DIMITROV, D.; HAUSOTTE, T.; WEGENER, K.; OVERMEYER, L.; VOLLERTSEN, F.; LEVY, G. N.. Laser based additive manufacturing in industry and academia. Cirp Annals, v.66, n.2, p.561-583, 2017. DOI: http://doi.org/10.1016/j.cirp.2017.05.011

SHEALY, T.. Do Sustainable Buildings Inspire More Sustainable Buildings?. Procedia Engineering, v.145, p.412419, 2017. DOI:

http://doi.org/10.1016/j.proeng.2016.04.008

SOLTAN, D. G.; LI, V. C.. A self-reinforced cementitious composite for building-scale 3D printing. Cement and Concrete Composites, v.90, p.1-13, 2018. DOI: http://doi.org/10.1016/i.cemconcomp.2018.03.017

SWAINSON, W. K.. Method, Medium and Apparatus for Producing Three-Dimensional Figure Product, U.S. Patent No. 4,041,476. Washington: U.S. Patent and Trademark Office, 1977.

WENG, Y.; RUAN, S.; LI, M.; MO, L.; UNLUER, C.; TAN, M. J.; QIAN, S.. Feasibility study on sustainable magnesium potassium phosphate cement paste for 3D printing. Construction and Building Materials, v.221, p.595-603, 2019. DOI:

http://doi.org/10.1016/j.conbuildmat.2019.05.053

WILHITE, R.; WÖLFEL, I.. 3D Printing for veterinary anatomy: An overview. Anatomia, Histologia, Embryologia, v.48, n.6, p.609-620, 2019. DOI: http://doi.org/10.1111/ahe.12502

A CBPC - Companhia Brasileira de Produção Científica (CNPJ: 11.221.422/0001-03) detém os direitos materiais desta publicação. Os direitos referem-se à publicação do trabalho em qualquer parte do mundo, incluindo os direitos às renovacões, expansões e disseminacões da contribuiç̃o, bem como outros direitos subsidiários. Todos os trabalhos publicados eletronicamente poderão preservam os direitos autorais, mas não têm permissão para a publicação da contribuição em outro meio, impresso ou digital, em português ou em tradução. 